\title{
Eosinophilic Granuloma Arising from the Sacrum: A Case Report
}

\author{
Yuji Kasukawa, Naohisa Miyakoshi, Michio Hongo, Shigeru Ando, Yoshinori Ishikawa, \\ Yoichi Shimada
}

Department of Orthopedic Surgery, Akita University Graduate School of Medicine, Akita, Japan.

E-mail: \{kasukawa, miyakosh, mhongo,nopposan, ishikaway, yshimada\}@doc.med.akita-u.ac.jp

Received October $4^{\text {th }}, 2011$; revised November $13^{\text {th }}, 2011$; accepted November $26^{\text {th }}, 2011$.

\begin{abstract}
Introduction: Osseous eosinophilic granulomas commonly occur in the skull, pelvis, vertebrae, mandible, and ribs. However, the eosinophilic granuloma in the sacrum is rare. Case presentation: We present the case of a 13-year-old Japanese boy, who presented with left low-back pain, and was diagnosed with eosinophilic granuloma arising in the sacrum. Computed tomography (CT) and magnetic resonance imaging demonstrated an osteolytic lesion with interruption of the cortex, and signal intensity changes at the left sacral body and wing. Histologic examination indicated an eosinophilic granuloma. Two years after CT-guided biopsy, the tumor had spontaneously healed completely, with no residual pain. Conclusion: The present case was eosiophilic granuloma arising from the rare site of sacrum. The tumor was completely remodeled two years after biopsy.
\end{abstract}

Keywords: Eosinophilic granuloma, Sacrum, Biopsy

\section{Introduction}

Eosinophilic granuloma is a benign tumor-like condition characterized by a clonal proliferation of Langerhans-type histiocytes in the bone or lung [1,2]. Eosinophilic granuloma of bone was initially described in 1940 [3,4], and its reported incidence is currently $0.05-0.5$ per 100,000 children per year, with $90 \%$ of tumors occurring under the age of 15 years [5-9]. Regarding the location of osseous eosinophilic granulomas, the flat bones are involved in $70 \%$ of tumors, and the long bones in 30\% [10]. Among the flat bones, eosinophilic granulomas predominantly affect the skull, pelvis, vertebrae, mandible, and ribs $[1,8]$. However, to the best of our knowledge, only one report has previously described this type of tumor in the sacrum [11]. We describe here a case of eosinophilic granuloma of the sacrum, which spontaneously healed completely after biopsy.

\section{Case Report}

A 13-year-old boy had a 2-month history of left lowback and buttock pain. He was referred to our institution when magnetic resonance imaging (MRI) at another hospital revealed abnormal changes in the sacrum. His left low-back and buttock pain was increased by lumbar extension. Neurologic examination on admission was nor- mal. Plain radiography showed an osteolytic change in the left sacral wing and sacral vertebral body (Figure 1(a)). Computed tomography (CT) images showed osteolytic low-density changes in the left sacral vertebral body extending to the sacral wing and sacro-iliac joint, with sclerotic changes around the osteolytic lesion. The cortex of the sacrum was interrupted at the sacro-iliac joint and the osteolytic lesion had a partly sclerotic margin (Figure 1(b)). MRI revealed slight high-intensity changes compared to muscle tissue on T1 weighted images, with strong uniform enhancement by gadolinium and nonuniform intensity changes on T2 weighted images (Figure 2). A technetium-99 $\mathrm{m}$ bone scan revealed uptake at the S1 vertebral body (Figure 3(a)). Based on these examinations, a malignant tumor such as Ewing's sarcoma or chronic osteomyelitis was considered as a differential diagnosis. Needle biopsy was performed under CT guidance through a posterior approach (Figure 3(b)). Histologic examination demonstrated mixed and dense cellular infiltrate including histiocytes with mildly irregular cell nuclei, eosinophils, neutrophils, and lymphocytes (Figure 3(c)). Histiocytes stained positive for CD68, which is found in the cytoplasmic granules and useful in diagnosing histiocytic diseases, on immunohistochemical staining (Figure 3(d)). These histologic findings led to a diagnosis of eosinophilic granuloma. The patient's pain 


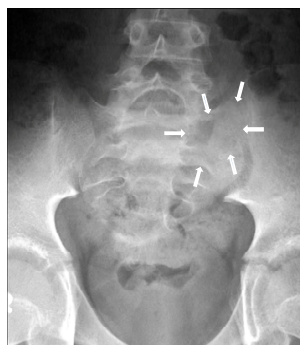

(a)

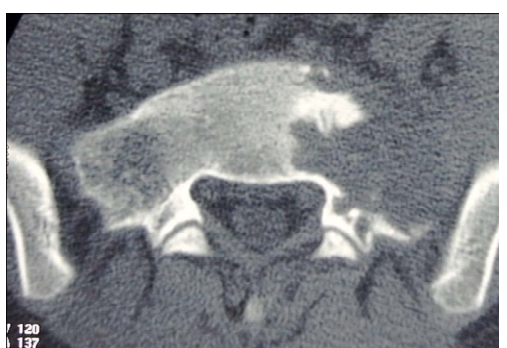

(b)

Figure 1. Plain radiograph and CT image before biopsy. (a) Anteroposterior plain radiograph of sacrum showing slightly defined osteolytic lesion on the left sacral wing (small arrows); (b) Axial CT demonstrates an osteolytic lesion with a partly sclerotic margin in the sacral body and the left sacral wing.

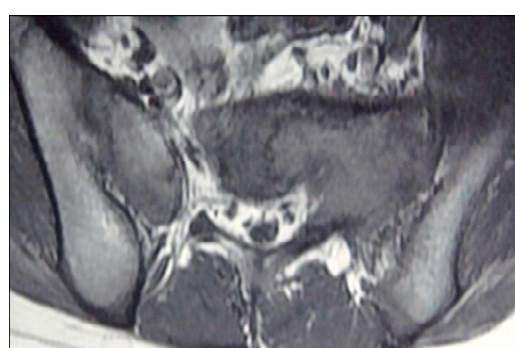

(a)

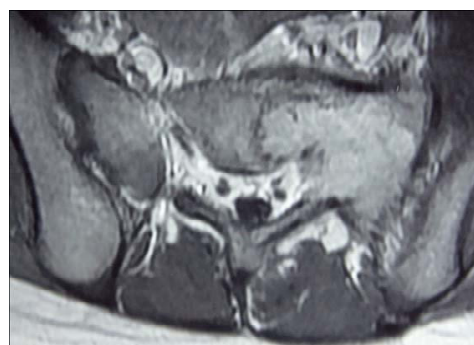

(b)

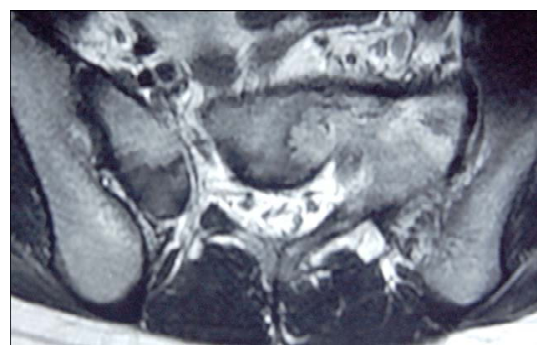

(c)

Figure 2. MRI of before biopsy.Lesion shows reduced signal intensity, which is isointense with muscle, in the axial T1weighted image (a) and is well-enhanced by gadolinium; (b) In the axial T2-weighted image; (c) the lesion has slightly higher intensity than the bone marrow of the ilium.

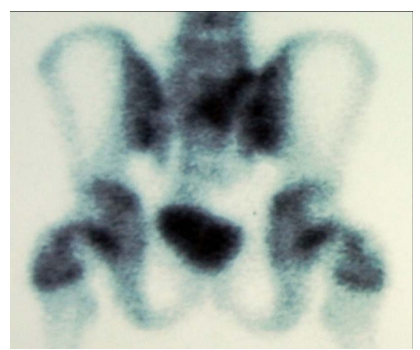

(a)

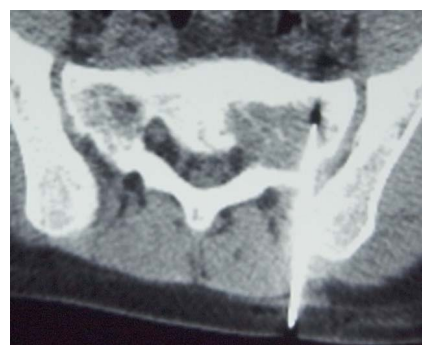

(b)

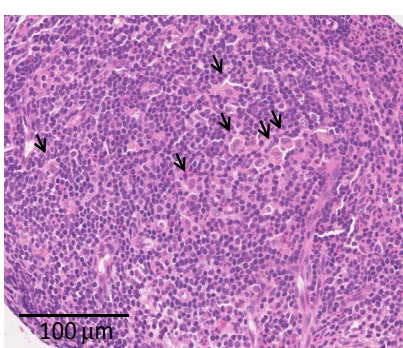

(c)

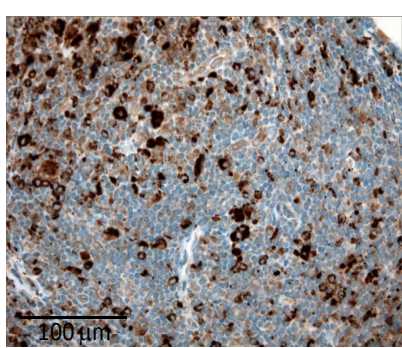

(d)

Figure 3. Bone scan before biopsy, CT image during biopsy, and histology. (a) Bone scan with technetium-99m showing increased uptake in the left sacral body and sacral wing. (b) Axial CT image during biopsy of the osteolytic lesion in the sacral wing. (c) Histologic examination demonstrating mixed and dense cellular infiltrate including histiocytes with mildly irregular cell nuclei (arrows), eosinophils, neutrophils, and lymphocytes. (d) Immunohistochemical staining showing CD68-positive staining of histiocytes.

gradually subsided following CT-guided biopsy, and CT and MRI images 3 months after biopsy showed an obvious decrease in the size of the osteolytic lesion. Two years after biopsy, his pain had completely disappeared, and the osteolytic lesion was not visible in CT images (Figure 4(a)), and appeared to be remodeled with fatty marrow in MR images (Figures 4(b) and 4(c)). Written informed consent was obtained from the patient for publication of this case report and accompanying images. A copy of the written consent is available for review by the Editor-in-Chief of this journal.

\section{Discussion}

The osseous lesions of eosinophilic granulomas appear as osteolytic, modestly destructive lesions of a flat bone, including the skull, mandible, ribs, and pelvis, in about $50 \%$ of cases [7]. Supraacetabular lesions represent the most common site of this tumor in the pelvis [12]. The iliac wings, iliac bone adjacent to the sacro-iliac joint, and the ischiopubic rami are other common sites for pelvic eosinophilic granulomas [12]. Eosinophilic granulomas occurring in the spine usually involve the vertebral body [6,12], showing characteristic symmetrical flattening 


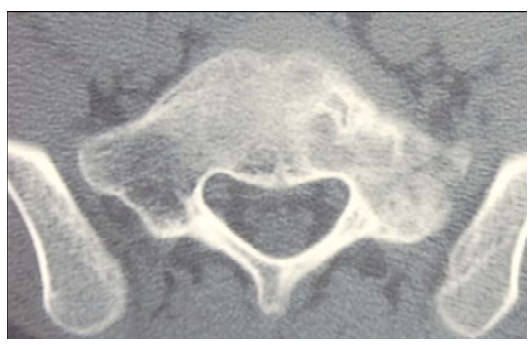

(a)

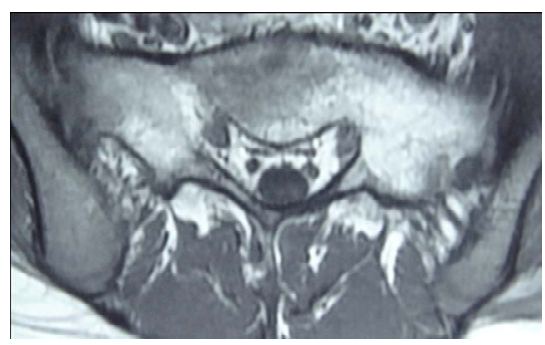

(b)

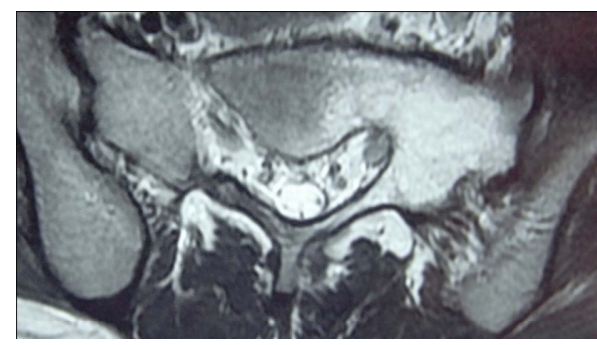

(c)

Figure 4. MRI and CT images two years after biopsy. (a) Axial CT image 2 years after biopsy demonstrating remodeled trabecular bone in the sacral body and wing, without interruption of cortex. The lesion demonstrated higher intensity compared to normal bone marrow in the axial T1-weighted (b) and T2-weighted images (c) 2 years after biopsy.

of the vertebra, known as "vertebra plana". The thoracic spine is the most common site, followed by the lumbar and cervical spine, respectively $[6,12]$. To the best of our knowledge, the present patient represents the first reported case of eosinophilic granuloma in the sacrum.

Although eosinophilic granuloma bone lesions are usually asymptomatic, osteolytic lesions sometimes cause fractures or pain as a result of swelling, deformity, and soft tissue components [6]. The chief complaint in the current case was low-back pain; however, the osteolytic lesion in the sacrum was poorly defined by plain radiography. MRI of the lumbar spine, however, revealed intensity changes in the sacrum. MRI thus provides a highly sensitive, but nonspecific, modality for detecting bone marrow involvement and soft tissue mass in eosinophilic granulomas [8].

Eosinophilic granulomas can be divided into acute and chronic phases, depending on their status $[6,8]$. The acute phase includes destructive, osteolytic lesions with poor margins, which are difficult to differentiate from malignant tumors such as Ewing's sarcoma, or acute osteomyelitis [8]. CT is useful for evaluating osseous eosinophilic granuloma lesions, and for determining the extent of cortical destruction and soft tissue involvement [8]. CT-guided biopsy is also useful for diagnosing eosinophilic granuloma as a cause of vertebral osteolysis or vertebra plana [9], and was helpful for diagnosing eosinophilic granuloma in the sacrum with cortical destruction in the present case.

Treatment options include aggressive resection, biopsy, combined chemotherapy and radiotherapy, and conservative treatment. Radiation therapy is associated with a risk of secondary malignancy [13] as well as effects on the pelvic viscera, and should be avoided, especially in children, because it may destroy the growth potential of the endochondral plates $[14,15]$. Ando et al. reported eosinophilic granulomas in the pelvis with different radiologic features and clinical courses [16]. They reported that osteolytic tumor lesions with sclerotic margins healed spontaneously after biopsy, while tumors with poor osteolytic margins progressed after biopsy. The present case was consistent with this previous report in that part of the osteolytic lesion in the sacrum revealed sclerotic margins on CT, and subsequently spontaneously healed completely. Surgical treatment, especially in adolescents, should be reserved for specific cases with neurologic defects or instability.

\section{Conclusions}

In conclusion, this patient represents the case of eosinophilic granuloma arising in the rare site of sacrum, which spontaneously and completely healed 2 years after biopsy. Biopsy is a better choice to decide a therapeutic strategy of the osteolytic tumor in the sacrum. If the tumor is eosinophilic granuloma, the tumor has a chance to heal spontaneously.

\section{REFERENCES}

[1] F. Plasschaert, C. Craig, R. Bell, W. G. Cole, J. S. Wunder and B. A. Alman, "Eosinophilic Granuloma: A Different Behavior in Children Than in Adults," Journal of Bone and Joint Surgery, Vol. 84, No. 6, 2002, pp. 870872. doi:10.1302/0301-620X.84B6.12585

[2] D. L. Muscolo, G. Slullitel, M. Ranalletta, L. A. AponteTinal and M. A. Ayerza "Spontaneous Remission of Massive Solitary Eosinophilic Granuloma of the Femur,” Journal of Pediatric Orthopaedics, Vol. 23, No. 6, 2003, pp. 763-765. doi:10.1097/01241398-200311000-00014

[3] S. Otani and J. G. Ehrlich, "Solitary Granuloma of Bone Simulating Primary Neoplasm,” American Journal of Pathology, Vol. 16, No. 4, 1940, pp. 479-490.

[4] L. Lichtenstein and H. L. Jaffe, "Eosinophilic Granuloma of Bone. With Report of a Case," American Journal of Pathology, Vol. 16, No. 5, 1940, pp. 595-604.

[5] P. Grundy and R. Ellis, "Hisiocytosis X: A Review of the Etiology, Pathology, Staging, and Therapy,” Medical and Pediatric Oncology, Vol. 14, No.1, 1986, pp. 45-50.

[6] R. David, R. A. Oria, R. Kumar, E. B. Singleton, M. M. Lindell, A. Shirkhoda and J. E. Madewell, "Radiologic Features of Eosinophilic Granuloma of Bone,” American Journal of Roentgenology, Vol. 153, No. 5, 1989, pp. 1021-1026. 
[7] M. A. Still, M. J. Kransdorf and K. O. Devaney, "Langerhans Cell Histiocytosis of Bone,” Radiographics, Vol. 12, No. 4, 1992, pp. 801-823.

[8] E. M. Azouz, G. Saigal, M. M. Rodriguez and A. Podda, "Langerhans' Cell Histiocytosis: Pathology, Imaging and Treatment of Skeletal Involvement,” Pediatric Radiology, Vol. 35, No. 2, 2005, pp. 103-115. doi:10.1007/s00247-004-1262-0

[9] K. B. Hoover, D. I. Rosenthal and H. Mankin, "Langerhans Cell Histiocytosis,” Skeletal Radiology, Vol. 36, No. 2, 2007, pp. 95-104. doi:10.1007/s00256-006-0193-2

[10] M. Monroc, H. Ducou le Pointe, S. Haddad, P. Josset and J. P. Montagne, "Soft Tissue Signal Abnormality Associated with Eosinophilic Granuloma. Correlation of MR Imaging with Pathologic Findings," Pediatric Radiology, Vol. 24, No. 9, 1994, pp. 328-332. doi:10.1007/BF02012118

[11] T. Cardon, E. Hachulla, R. M. Flipo, P. Chastanet, C. Rose, X. Deprez, B. Delcambre and B. Devulder, "Percutaneous Vertebroplasty with Acrylic Cement in the Treatment of a Langerhans Cell Vertebral Histiocytosis,” Clinical Rheumatology, Vol. 13, No. 3, 1994, pp. 518521. doi:10.1007/BF02242955
[12] J. C. Leonidas, “Langerhans’ Cell Histiocytosis. In Radiology: Diagnosis, Imaging, Intervention,” Edited by J. M. Taveras and J. M. Ferrucci, Lippincott, Philadelphia, 1990, pp. 1-9, 12.

[13] L. Johansson, L. G. Larsson and L. Damber, "A Cohort Study with Regard to the Risk of Haematological Malignancies in Patients Treated with X-rays for Benign Lesions in the Locomotor System," Acta Oncologica, Vol. 34, No. 6, 1995, pp. 721-726. doi:10.3109/02841869509127178

[14] N. E. Green, W. W. Robertson Jr and A. W. Kilroy, "Eosinophilic Granuloma of the Spine with Associated Neural Deficit: Report of Three Cases,” Journal of Bone and Joint Surgery, Vol. 62, No. 7, 1980, pp. 1198-1202.

[15] M. J. Silberstein, M. Sundaram, B. Akbarnia, A. Luisiri and M. McGuire, "Eosinophilic Granuloma of the Spine," Orthopedics, Vol. 8, No. 2, 1985, pp. 267-274.

[16] A. Ando, M. Hatori, M. Hosaka, Y. Hagiwara, A. Kita and E. Itoi, "Eosinophilic Granuloma Arising from the Pelvis in Children: A Report of Three Cases," Upsala Journal of Medical Sciences, Vol. 113, No. 2, 2008, pp. 209-216. doi:10.3109/2000-1967-230 